\title{
Taking steps towards the European medical specialist in ENT
}

During the " 1 st meeting of the European Academy of ORL-HNS" (Congress Center Rosengarten, Mannheim, Germany, June 27-30, 2009), the European Board Examination in ORL-HNS will take place for the first time.
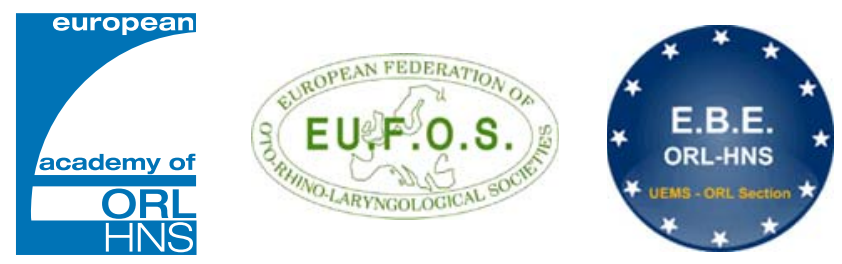

The European Examination Board in Otorhinolaryngology (EBEORL) was created by the UEMS ORL Section and Board in October 2008, in order to harmonize the knowledge achieved by Otorhinolaryngologists throughout Europe. The aim of this independent supra-national (European) Board is to set up a quality standard for all of Europe, in order to allow free movement of specialists between member countries.

Candidates of any nationality shall be eligible to sit the European exam, provided they have submitted all documents necessary and have been approved by the Board. The application procedure runs from Monday, March 2 until Friday, May 29, 2009.

Candidates who pass the European Board Exam in ORL who are certified specialists in an UEMS member state may call themselves "Fellow of the European Board of Otorhinolaryngology". Other successful candidates will receive a "Diploma of the European Board of Otorhinolaryngology".

Details on the exam and the application procedure can be found at http://www.ebeorl-hns.org.

Interest is high in the Mannheim meeting: more than 800 participants from $60+$ countries had registered for the congress by February 2009, and more than 400 abstracts were submitted.

Putting to use the valuable experiences gained while devising the scientific programme of 2007s hugely successful "6th EUFOS Congress" in Vienna, the programme structure for the Mannheim meeting has been further refined to focus even more on education and lively interaction. After all, the main task of the European Academy of ORL-HNS is the training and continuing education of ENT doctors in different stages of their careers (for more information on the EAORL-HNS, please visit http://www. eaorl-hns.org).

The meeting - the largest of its kind in Europe-will present an outstanding opportunity for Otorhinolaryngologists of all nations and from all sub-specialities to come together in a relaxed friendly environment and to exchange their ideas and expertise. With its emphasis on Instructional Courses and Round Tables, it will offer a wide range of scientific as well as practical education to doctorsin-training and young scientists from all over the world.

50 Instructional Courses for all fields have already been confirmed, with topics ranging from OSAS and reflux disorders to oncology, from modern face lifting techniques and Botox to vocal cord dysfunction, from auditory implants and stapes surgery to chronic rhinosinusitis and septoplasty.

In case you haven't registered yet, we cordially invite you to be part of this "1st Meeting of the EAORL-HNS". The deadline for regular registration is June 5, 2009. After that date, the on-site fee applies.

All information on the congress can be found at http:// www.eaorl-hns2009.com. 